\title{
Research on the Scheduling Algorithm Based on the Resource Transport Path of the Contingency Cellular Network
}

\author{
Xiaoguang $\mathrm{Li}^{*}$ and Tao Yang
}

Department of Electrical Engineering and Automation, Luoyang Institute of Science and Technology, Luoyang, Henan, 471023, China

\begin{abstract}
Considering the relative distance among the base stations, the time and the relief profits in the base stations, the paper proposes optimal scheduling models CCNDS-AC and CCNDS-UC which are fit for the Resource Transport Path of the Contingency Cellular Network with the topology-tree structure. When a large natural disaster happens, time is rather urgent and the admissible computing time is quite short, two kinds of the fast heuristic algorithms are proposed: DS-ACG and DS-UCB so that a group of the construction scheduling order which is approximating the optimal solution is obtained in the short time. The experimental results show that the proposed algorithm efficiency can effectively improve $16 \%$ so that the maximal total relief benefits can be reached.
\end{abstract}

Keywords: CCN, the Heuristic Algorithm, the optimal scheduling model.

\section{INTRODUCTION}

\subsection{The Influence of the Large-Scale Disasters}

When the large disasters are coming, the disaster areas are so turbulent that the disaster scenes are difficult to be described. The relief actions are urgently needed and the profit of the relief determines many people's life. Integrating the experience of all kinds of the natural disasters, several important factors influencing the relief profit in the large natural disasters are summarized as follows:

The traffic paralysis in the disaster areas: The roads inside or outside the disaster areas will be damaged so that the traffic in the disaster areas are paralyzed and the air traffic may also be interrupted by the weather or other factors. The survivals may also be packed by a large of the volunteering relief cars.

The communication network paralysis: The communication networks in the fixed network and the mobile phones are nearly paralyzed. The survival communication networks will be congested with a large number of the care calls so that the communication networks cannot be adopted for the relief.

A serious lack of the specialized relief personnel: The number of the specialized relief personnel is very not enough, especially in the beginning of the disaster. Therefore, a majority of the local volunteers must be mobilized to take part in the relief.

The failure of the administrative command system: Each administrative command system may be damaged so that the communicating organization is paralyzed.

\subsection{The CCN}

A large part of the mobile phone base station which interrupts the service will stop being operated for the interruption of the power and the fixed network line connecting with the end, while the base station is not damaged. CCN [1-3] adopts the long-distance $\mathrm{WiFi}$ online (or other accessible wireless radio technology) to connect with the base station construction of disaster emergency communication system which is survived and whose service is interrupted (as shown in the Fig. 1). The users of the mobile phones in the disaster can use the mobile phones whose signal is interrupted as the communication of the relief [4]. For example, If the mobile phones within the range of the base station are used or used as the wireless inter-phones, a greater convenience can be provided to the relief. In addition, all available resources should be used in the relief as soon as possible under the limitation of transmitting the resources $[5,6]$.

The main equipment of the $\mathrm{CCN}$ is Contingency Recovery Package, including the generator, the fuel equipment, the wireless communication equipment and its core equipment EC Module [7-9]. All of theses equipment are stored in the center of national relief-prevention or the company of the mobile phones. When the disaster happens, the relief goods should be delivered into the disaster areas through the air drop or helicopter method (the base stations is always under the condition of the inconvenient transportation) [10]. The base stations can adopt the wireless communication equipment to connect with each other and the jump connection method to reply the online among the core networks so that a part of communicating function can be recovered and the users of the mobile phoned in the disaster areas can be supported. The system has several advantages: 1 . Reusing the original mobile communicating base stations can reduce the cost and cover a wide range. 2 . The topology of the original $\mathrm{CCN}$ is designed sophistical and located in the high position. 


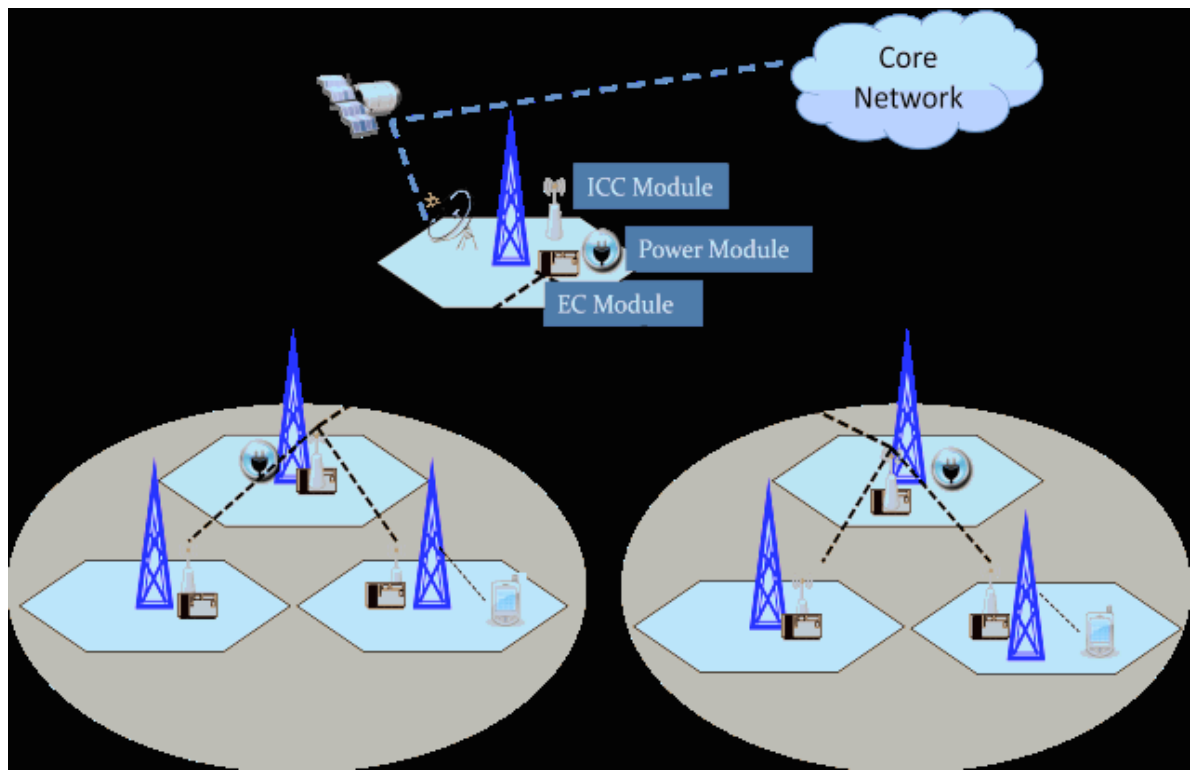

Fig. (1). The CCN general frame.

3 . The mobile phones without being refitted are so popular that they can be directly used, and a large number of money cannot be paid to buy the mobile phones. 4 . The users of the mobile phones are not to be trained for everybody knows how to use it. 5. During the coming of the disasters, people may carry a mobile phone when they escape from their homes or are in trouble unfortunately.

\subsection{The Scheduling of the $\mathrm{CCN}$}

After the large natural disasters happen, the different disaster degrees in every area are caused so that the $\mathrm{CCN}$ must be initiated. After the planning of the $\mathrm{CCN}$ topology is improved, the $\mathrm{CCN}$ topology must be quickly built. During the building of the CCN topology, the transport vehicles and the number of the building engineering personnel are so limited that the resources can not be reached once, and the needed resources should be transported by many times helicopter, car and personal transport in turn so that the $\mathrm{CCN}$ topology can be built. How to stipulate an order of the restoring scheduling is according to the degrees of the disasters in each service area of the base station, that is, the building order of the base station, and the total profit of the relief is the maximum. The profit brought by restoring a base station is a time function. The function is defined by the relief commanding center and changed with the passage of the time. If the base station is restored more early and used in the relief, the profit is higher. If the topology of $\mathrm{CCN}$ is confirmed before the scheduling and belongs to a tree topology, it is called the Forwarding Tree $[11,12]$. Therefore, the problem of restoring the scheduling can be simply described as follows: Aiming to the defined transmitting topology within the limited time, a group of restoring order in the base station should be found out so that the total profit of all base stations can be maximized [13].

\section{THE RESOURCE TRANSPORTATION ROUTE}

The paper proposes two problem models: the first problem model is CCN Deployment Scheduling Antecedence Constrained (CCNDS-AC) [14]. The model is initiated from the networking platform, transmits the topology in terms of the $\mathrm{CCN}$ tree structure and priory builds the father nodes. After a certain base station is built, it can be put into service. The second problem model is CCN Deployment Scheduling Unconstrained (CCNDS-UC). The deployment scheduling of the base station is done with eliminating the limited conditions of the tree-structure transmitted topology considers more possibilities of the deployment scheduling. If the father nodes of a certain base station are not put into the deployment for the unfixed factors caused by the father nodes which begin to be deployed so that they can not be put into the service immediately when the base station is deployed completely. However, when the father nodes are restored and can be put into the service, it can also be put into the service.

\subsection{The CCNDS-AC Problem Model}

The concept of CCNDS-AC is initiated from the networking platform and accorded with the rules of priory deploying the father nodes to deploy in terms of the tree structure of the CCN topology, road network and other related information. If the father nodes of the node are not deployed, they can not be within the range of the considered candidate node lists. When the deployment of a signal base station is finished, it can immediately serve the base station and cover the disaster areas, namely, the relief profit can be obtained during the period of finishing the deployment. The CCNDSAC problem model is described as follows:

Given a forwarding tree $T(V, E), D, P, S$ where

$1 \mathrm{~V}=\{\mathrm{vi} \mid \mathrm{i}=1,2, \ldots, \mathrm{n}\}$ is the set of survival base stations and isolated base stations.

1v1 is the root node that has an external link (survival base station).

$1 \mathrm{E}=\{$ eij|eij is the link of (vi,vj) and $v i, v j \in \mathrm{V}\}$

$1 \mathrm{D}=\{\mathrm{dij} \mid \mathrm{dij}$ is the traveling time of $(\mathrm{vi}, \mathrm{vj})$ and $\mathrm{vi}, \mathrm{vj} \in \mathrm{V}\}$

$1 \mathrm{~S}=\{\mathrm{sk} \mid \mathrm{sk}$ is a permutation of $\mathrm{V}, \mathrm{k}=1, \ldots, \mathrm{n} !\}$ is the set of $\mathrm{CCN}$ construction schedules. 


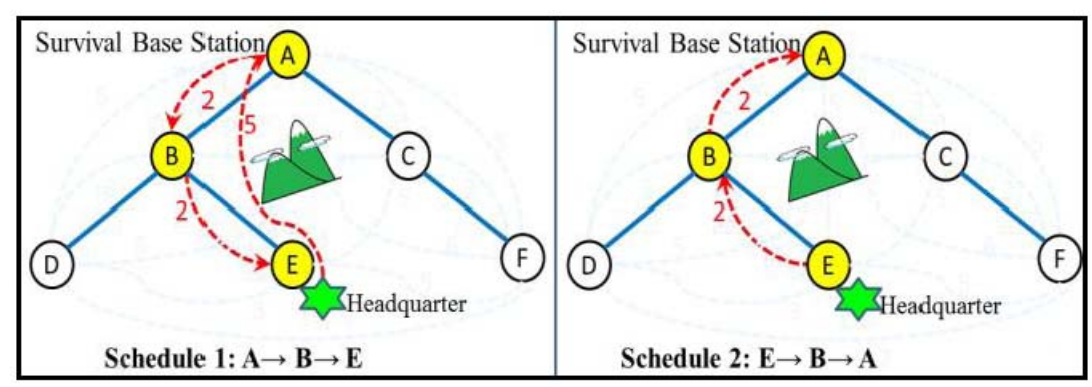

Fig. (2). The example explanation of the prior father nodes deployment.

$1 \mathrm{C}=\{\mathrm{ci}(\mathrm{sk}) \mid \mathrm{ci}(\mathrm{sk})$ is the time at which node vi has be repaired in the schedule sk and $v i \in \mathrm{V}, \mathrm{sk} \in \mathrm{S}\}$

$1 \mathrm{P}=\{\operatorname{pi}(\mathrm{t}) \mid \mathrm{i}=1, \ldots, \mathrm{n}, \mathrm{t} \in \mathrm{Z}+\}, \mathrm{pi}(\mathrm{t})$ is the profit of $v i$, if node vi is constructed at time $t, t=c i(s k)$ and $s k \in S$.

The CCN deployment scheduling antecedence constrained problem is to find $s k \in \mathrm{S}$, such that Maximize $\Sigma \mathrm{vi}(\mathrm{t})=\Sigma \mathrm{vi} p(\mathrm{ci}(\mathrm{sk}))$

Subject to vi must be constructed before $v j$, if vi is the antecedence of $\mathrm{vj}$.

\subsection{The CCNDS-UC Problem Model}

The CCNDS-AC is initiated from the networking platform and accorded with the rules of priory deploying the father nodes to deploy, which can make the moving lines rather repeated and cost so much time that the loss of the relief profit is caused during the deployment of the scheduling. The Fig. (2) shows that Schedule 1 is initiated from the relief Headquarter to the networking platform and cost 5 times for priory deploying the father nodes, and then $\mathrm{B}$ and $\mathrm{E}$ are deployed consequently and its total cost time is 9 . While Schedule 2 is initiated from the E near to the relief Headquarter and the $\mathrm{B}$ and $\mathrm{A}$ should be deployed with the networking platform, its total cost time is 4 . During the deployment of $\mathrm{A}, \mathrm{B}$, and $\mathrm{E}$, the limitation of CCNDS-AC not only costs much deployment time, but also wastes the vehicle resources and influences the relief profit.

The proposed CCNDS-UC expects to improve the above problems and obtain a greater relief profit. The problem model eliminates the limited condition of the tree structure for it does not force the order deployment from up to down. Although the deployment of a signal base station can not be immediately put into the service, it can be put into the service as long as the father nodes are put into the service. In this way, much delivering time can be saved. After finishing the deployment of the topology, the maximum relief profit can be reached. The CCNDS-UC problem model is described as follows:

Given a forwarding tree $\mathrm{T}(\mathrm{V}, \mathrm{E}), \mathrm{D}, \mathrm{P}, \mathrm{S}$ where

$11 \mathrm{~V}=\{\mathrm{vi} \mid \mathrm{i}=0,1,2, \ldots, \mathrm{n}\}$ is the set of survival base stations and isolated base stations.

$11 \mathrm{v} 1$ is the root node that has an external link (survival base station).

$11 \mathrm{v} 0$ is the $\mathrm{CCN}$ headquarter

$1 \mathrm{E}=\{$ eij|eij is the link of $(v i, v j)$ and $v i, v j \in V\}$

$1 \mathrm{D}=\{\mathrm{dij} \mid \mathrm{dij}$ is the traveling time of $(\mathrm{vi}, \mathrm{vj})$ and $v i, v j \in \mathrm{V}\}$
$1 \mathrm{~S}=\{\mathrm{sk} \mid \mathrm{sk}$ is a permutation of $\mathrm{V}, \mathrm{k}=1, \ldots, \mathrm{n} !\}$ is the set of $\mathrm{CCN}$ construction schedules.

$1 \mathrm{C}=\{\mathrm{ci}(\mathrm{sk}) \mid \mathrm{ci}(\mathrm{sk})$ is the time at which node vi has be repaired in the schedule sk and $v i \in \mathrm{V}, \mathrm{sk} \in \mathrm{S}\}$

$1 \mathrm{P}=\{\mathrm{pi}(\mathrm{t}) \mid \mathrm{i}=1, \ldots, \mathrm{n}, \mathrm{t} \in \mathrm{Z}+\}, \mathrm{pi}(\mathrm{t})$ is the profit of $v i$, if node vi is constructed at time $\mathrm{t}, \mathrm{t}=\mathrm{ci}(\mathrm{sk})$ and $\mathrm{sk} \in \mathrm{S}$.

The CCN deployment scheduling antecedence constrained problem is to find $s k \in \mathrm{S}$, such that Maximize $\Sigma \mathrm{vi}(\mathrm{t})=\Sigma \mathrm{vi} p(\mathrm{ci}(\mathrm{sk}))$

\section{ALGORITHM DESIGN}

CCNDS-AC and CCNDS-UC belong to the NP-Hard problems. A group of the optimal solution within the limited time can not be found out for the relief activity is very urgent; time is rather valuable and the range of the disaster areas so large that many numbers of the base stations must be deployed. Therefore, a rapid heuristic algorithm for the two problem models is designed. Therefore, the CCN deployment scheduling approximating to the optimal solution can be computed within the short time.

\subsection{The DS-ACG Algorithm}

Deployment Scheduling Antecedence Constrained Greedy Algorithm (DS-ACG) is aiming to the first problem model CCNDS-AC. The algorithm search for a node which can obtain the maximum relief profit in the unit time as the next restoring node until all nodes in the $\mathrm{CCN}$ topology can be restored under the condition of the father nodes prior deployment. The DS-ACG pseudo code has been shown in the Fig. (3).

\subsection{The DS-ACG Algorithm}

Deployment Scheduling Unconstrained Backtrack Algorithm (DS-UCB) is aiming to the first problem model CCNDS-UC. The algorithm uses the neighboring node to restore the deployment under the limited condition of deleting the father nodes prior deployment. In this way, much time and many delivering resources can be saved. If it just adopts to the greedy algorithm, the relief profit may not be obtained and the neighboring nodes can not be selected. Therefore, the heuristic algorithm different from the greedy algorithm is designed and the Backtracking mechanism should be matched. Furthermore, the path and the relief profit of the neighboring nodes should be considered, and the scheduling should be changed until all nodes in the CCN topology are restored. The DS-UCB pseudo code has been shown in the Fig. (4). 


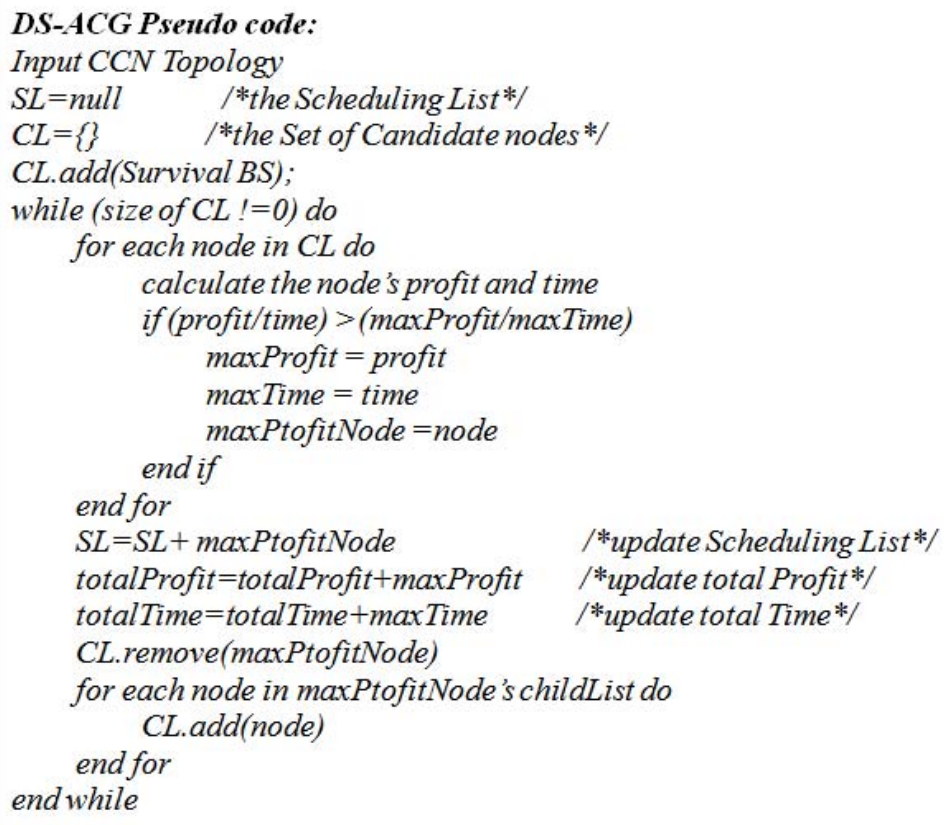

Fig. (3). The DS-ACG pseudo code.

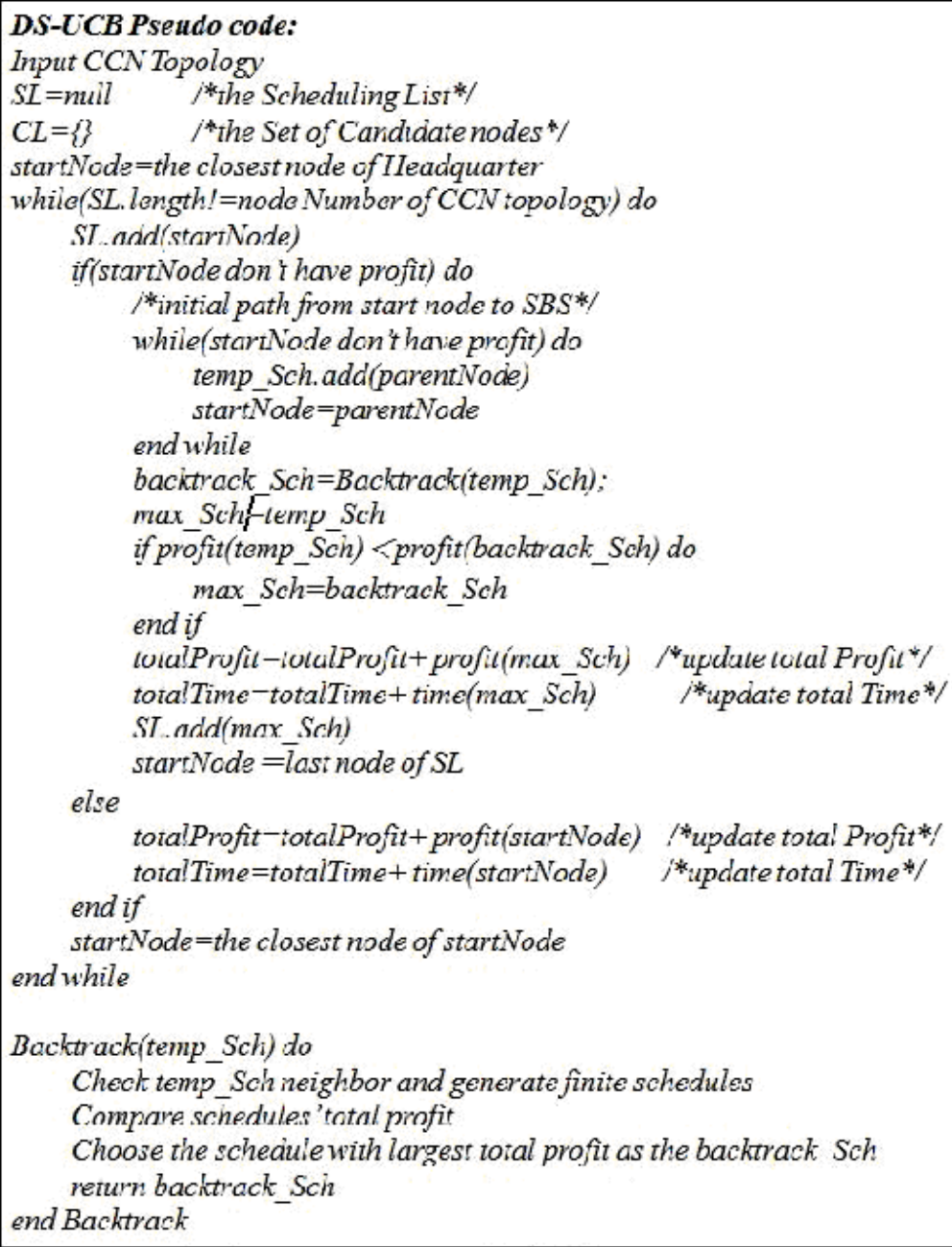

Fig. (4). The DS-UCB pseudo code. 


\section{EXPERIMENTAL RESULTS}

The random method is adopted to produce a large number of CCN topology. The DS-ACG, the DS-UCB, the proposed DS-G and the brute-force operation should be large scale and small scale experimented for obtaining the deployment scheduling of the CCN.

The large-scale cases in the experiment should be done. When the number of the base stations changed, the optimal solution cannot be computed in the short time. Therefore, the pseudo optimal solution and the pseudo worst solution are adopted to estimate the total relief benefit and the total relief time under the experiment. The estimated indexes of the experimental results should be estimated:

Total Profit: The total benefit is obtained from the restoring nodes in the algorithm.

Total Time: After the whole $\mathrm{CCN}$ is restored; the total time means the total cost time during deploying the scheduling.

Original Deviation: Original Deviation means that the optimal solution is compared with the total benefit, as shown in the formula 1 .

Original Deviation $=\frac{\text { Algorithm solution }}{\text { Pseudo optimal solution }}-1$

Normalize Deviation: As the experimental cases are randomly produced by the random numbers, each experiment is independent and not related. The obtained profit must be through the Normalization, as shown in the formula 2 (Table 1).

Normalize Deviation $=$

Algorithm solution - pseudo optimal solution

Pseudo optimal solution - Pseudo worst solution

Experimental Results

The DS-ACG and the DS-UCB are proposed in the paper according to the relief scheduling and the factors approximating to the actual situation. The proposed algorithm DS-G does not consider the relative distance among the base sta- tions so that the total relief time is higher than the proposed DS-ACG and DS-UCB, and its total relief profit is lower than the proposed DS-ACG and DS-UCB.

Table 1. Experiment parameters.

\begin{tabular}{|c|c|}
\hline Parameters & Range of Values \\
\hline \hline Initial Profit & Uniform $(30,120)$ \\
\hline Traveling Time & Uniform $(0.5,10) \mathrm{hr}$ \\
\hline Forwarding Tree Size & 50 \\
\hline Slope S1 & Uniform $(-1,0)$ \\
\hline Slope S2 & Uniform $(-1,0)$ \\
\hline Turning Point & Uniform $(50,100) \mathrm{hr}$ \\
\hline
\end{tabular}

The algorithm DS-UCB should develop its advantages and deploy the nodes whose relief profit is very high so that the total relief time can be saved much, that is, 19.4 percent of the total relief time can be saved averagely so that the total relief profit can have a good effect, and its average profit can improve about $16.66 \%$, as shown in the Fig. (5). While the algorithm is limited by the prior deployment of the father nodes, the number of the deployment scheduling increase, its total relief profit is worse, but can can be more approximating to the pseudo optimal solution than the algorithm DS-G. Compared with the DS-G, the total relief proficient in the DS-ACG is over 52.67\% (as shown in the Fig. 6).

\section{SUMMARY}

When the large-scale natural disasters happen, the heavy casualties are caused and the communication system is often palatalized so that the relief activity is hindered. The effective operated communication system is the critical factors whether the transmission of the disastrous situation, the resource scheduling, and the relief activity can be smooth. Although the Wireless Radio is adopted to complete the function, the normal online of the base station can not be con-

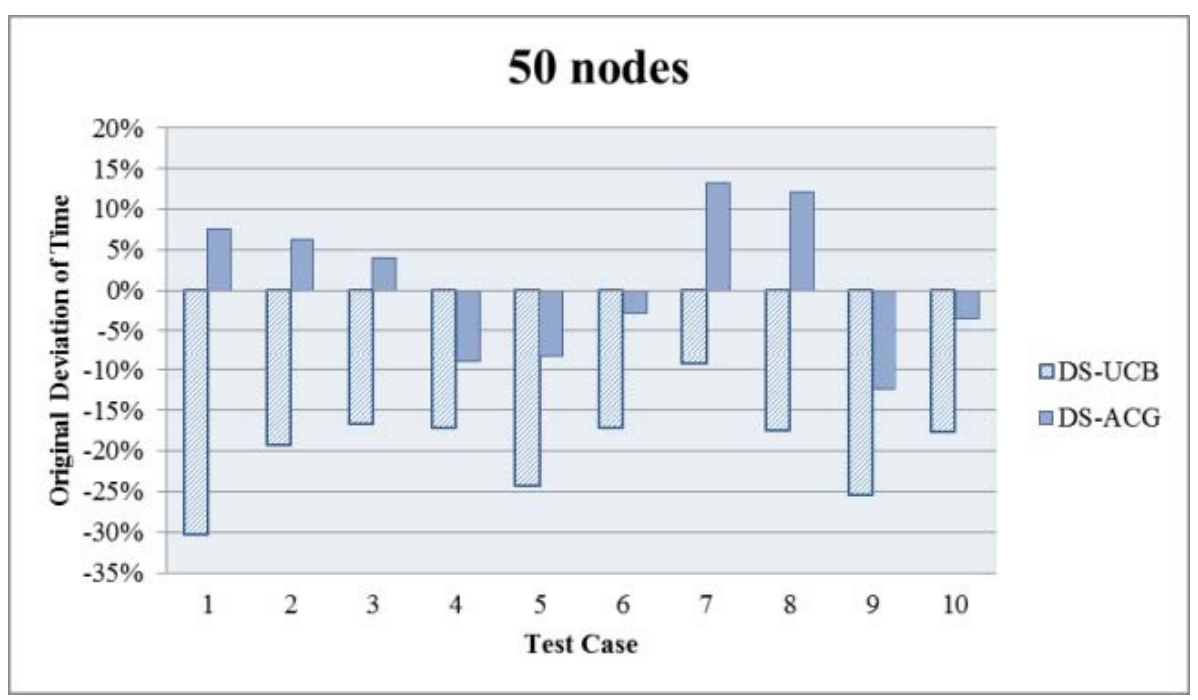

Fig. (5). Original deviation of time (50 nodes). 


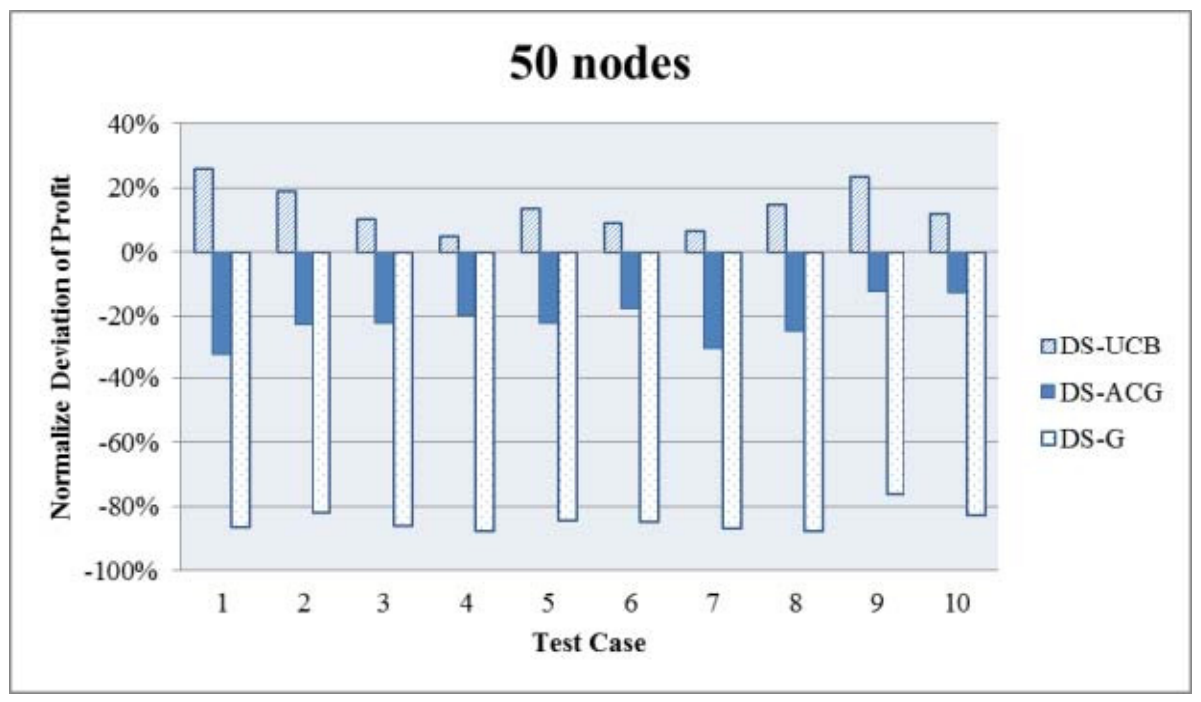

Fig. (6). Normalize deviation of profit (50 nodes).

nected with the outside so that the temporary mobile communication network which is called CCN is built. As the disastrous situation in each area is not completely the same so that the deployment order of the CCN should consider the following factors, such as the priorities of the disaster areas, the urgency of time and others. After the planning of the $\mathrm{CCN}$ topology is finished, the deployment scheduling of the base station should be planned according to the CCN topology, the degrees of the disastrous situation and the relative distance among the base stations so that the maximum total relief profit can be reached.

According to the reference [6], two optimal scheduling models fitted for considering resources delivering path of the $\mathrm{CCN}$ topology tree structure of are proposed by considering the relative distance between the base stations, the time and the relief profits so that the maximum of the total relief profit can be obtained. These models can offer the relief commanding unit system to solve the problem of the CCN deployment scheduling. The obtained relief profit can be defined by the relief units in terms of the disastrous situations. The proposed deployment scheduling models and the deployment scheduling algorithms can determine the deployment priority of the base stations in the $\mathrm{CCN}$ topology.

The problem of the CCN deployment scheduling is the more complex Single Machine Scheduling Problem. The paper proves that the problem belongs to the NP-Hard problem. When a large-scale natural disaster happens, time is rather urgent and the admissible computing time is quite short, two kinds of the fast heuristic algorithms are proposed: DS-ACG and DS-UCB so that a group of the construction scheduling order which is approximating the optimal solution is obtained in the short time. The DS-ACG algorithm can obtain the relief profits and the relative distance time among the base stations in terms of the CCN topology, the completion of the base station deployment. The deployment of the scheduling order of the maximum total relief profits should be found out when the father nodes are prior to the sub nodes. In order to solve the waste of the delivering resources, the reduction of the relief profits during the deployment of the father nodes which are priorer to the sub nodes, the DS-UCB algorithm is proposed. The algorithm deletes the limitation of the father nodes prior deployment and adds the neighboring nodes whose relief profits are very high into the deployment scheduling so that more delivering resources can be saved, the total relief time can be reduced and the total relief profits can be improved. The results show that the proposed algorithm DS-ACG and the DS-UCB are superior to the DS-G. In addition, the DS-UCB should develop its advantages and add the neighboring nodes whose relief profits are very high into the deployment scheduling so that the total relief time can be saved much. Compared with the pseudo optimal solution, the total relief time can be averagely saved about $19.4 \%$ and the average efficiency of the total relief profits can improve about $16.66 \%$ from the estimation index of the Original Deviation.

The models proposed in the paper have the improved space. For example, the deployment scheduling order operated by all kinds of different deployment teams, the clustering problem of the limited number of the carrier chicle load CPR and all kinds of different algorithms (genetic algorithms, traveled problems and related problems) should be improved. In addition, when the $\mathrm{CCN}$ topology has many networking platforms, namely, it is not only a single tree structure, the deployment scheduling order operated by all kinds of different deployment teams is an important researching issue. The CCN topology deployment should be planned and completed as soon as possible within the limited time. Moreover, the $\mathrm{CCN}$ should play an important role in helping the communications of the relief activities so that more life can be saved.

\section{CONFLICT OF INTEREST}

The authors confirm that this article content has no conflict of interest.

\section{ACKNOWLEDGEMENTS}

Science of Technology Research of Foundation Project of Henan Province Education Department under Grant Nos. 2014B520099, and Natural Science and Technology Research of Foundation Project of Henan Province Department of Science under Grant Nos. 142102210471. 


\section{REFERENCES}

[1] C. Zou, J. Wan, M. Chen, and D. Li, "Simulation modeling of cyber-physical systems exemplified by unmanned vehicles with WSNs navigation," In: Proceedings of the $7^{\text {th }}$ International Conference on Embedded and Multimedia Computing Technology and Service, Gwangju, Korea, September 2012, pp. 269-275.

[2] Y. Bai, W. Du, Z. Ma, C. Shen, Y. Zhou, and B. Chen, "Emergency communication system by heterogeneous wireless networking," In: Proceedings of IEEE Wireless Communications, Networking and Information Security (WCNIS), June 2010.

[3] J. Wan, H. Yan, Q. Liu, K. Zhou, R. Lu, and D. Li, "Enabling cyber-physical systems with machine-to-machine technologies," Ad Hoc and Ubiquitous Computing, vol. 13, no. 3/4, pp. 187-196, 2013.

[4] K. T. Phan, T. L. Ngoc, M. V. S. Schaar, and F. Fu, "Optimal scheduling over time-varying channels with traffic admission control: structural results and online learning algorithms, " IEEE Transactions on Wireless Communications, vol. 12, no. 9, pp. 4434-4444, 2013.

[5] S. F. Xu, G. Zhu, C. Shen, and Y. Lei, "Delay-aware fair scheduling in relay-assisted high-speed railway networks, " In: Proceedings of the International Conference on Communications and Networking in China, pp. 17-21, 2013.

[6] W. Luo, X. Fang, M. Cheng, and Y. Zhao, "Efficient multiplegroup multiple-antenna (MGMA) scheme for high-speed railway viaducts," IEEE Transactions on Vehicular Technology, vol. 62, no. 6, pp. 2558-2569, 2013.

[7] B. L. Niu, V. W. S. Wong, and R. Schober, "Downlink scheduling with transmission strategy selection for multi-cell MIMO systems“",
IEEE Transactions on Wireless Communications, vol. 12, no. 2, pp. 736-747, 2013.

[8] H. Azogu, "Privacy-preserving license plate image processing, " In Proceedings of the $2^{\text {nd }}$ IEEE Workshop on Multimedia Communications and Services, IEEE GlobeCom, 2011, Houston, TX, 5-9 December 2011.

[9] J. S. Huang, Y.N. Lien and Y.C. Huang, "Network Topology Planning for Contingency Cellular Network", In: Proceedings of $17^{\text {th }}$ Mobile Computong Workshop, Aug. 2012, Taiwan.

[10] Y. Han and L. Shi, “ Optimization Based Method for Supply Location Selection and Rounting in Large-Scale Emergency Material Delivery", IEEE Transactions on Automation Science and Engineering, vol. 8, no. 4, pp. 683-693, Oct. 2011.

[11] Y.N. Lien, L. C. Chi, and Y. S. Shaw, "A Walkie-Talkie-Like Emergency Communication System for Catastrophic Natural Disasters," In: Proceedings of $10^{\text {th }}$ International Symposium on Pervasive system, Algorithm and Networks, (ISPAN), Dec. 2009, pp. 309-314.

[12] Z. Lu and S. Luo, "Emergency Supplies' Two Transport Scheduling Model", In: International Conference on Transportation, Me chanical, and Electrical Engineering (TMEE), Dec. 2011.

[13] Z. Shao, Y. Liu, Y. Wu, and L. Shen, "A Rapid and Reliable Disaster Emergency Mobile Communication System via Aerial Ad Hoc BS Networks," In: Proceedings of IEEE Wireless Communications, Networking and Mobile Computing (WiCOM), Sept., 2011.

[14] Y. Ran, "Considerations and Suggestions on Improvement of Communication Network Disaster Countermeasures after the Wenchuan Earthquake", IEEE Communications Magazine, vol. 49, no. 1, pp. 44-47, Jan. 2011.

(C) Li and Yang; Licensee Bentham Open.

This is an open access article licensed under the terms of the Creative Commons Attribution Non-Commercial License (http://creativecommons.org/licenses/by-nc/3.0/) which permits unrestricted, non-commercial use, distribution and reproduction in any medium, provided the work is properly cited. 